\title{
Study of Antimicrobial Resistance Profile of Coagulase Negative Staphylococcus Species Isolated from the Oral Cavity of Indian Cobra (Naja naja)
}

\section{Soumya Ranjan Khatua ${ }^{1 *}$, Rajashree Mishra ${ }^{1}$, Indramani Nath ${ }^{2}$, Debiprasanna Das ${ }^{3}$, Bhabani Shankar Satapathy ${ }^{1}$, Kailash Singh Bisht ${ }^{1}$ and Iswar Anand Senapati ${ }^{1}$}

${ }^{1}$ Department of Veterinary Microbiology, ${ }^{2}$ Department of Veterinary Surgery \& Radiology, ${ }^{3}$ Department of Veterinary Pathology, College of Veterinary Science and Animal Husbandry, OUAT, Bhubaneswar, India

*Corresponding author

\section{A B S T R A C T}

\section{Keywords}

Naja naja, Mouth, Staphylococcus, Biofilm

\section{Article Info}

Accepted:

07 August 2020

Available Online:

10 September 2020
Snake bite is a major public health concern which can cause bacterial infection along with envenomation. The infection may be due to the bacteria present in mouth of venomous or nonvenomous snake which may contaminate the bite site during biting. In the present study coagulase negative Staphylococcus species from the oral cavity of two Indian cobras were isolated and identified and were screened for its biofilm forming ability using bentonite clay as a substrate. Various methods like Congored agar assay and tissue culture plate method were also used for quantitative estimation of biofilm formation. Oral swabs were collected from a 5year old female and 8year old male Indian cobra in an aseptic manner after observing the guidelines of capturing the wild snakes by professional environmentalists. The swab samples were processed as per routine microbial procedure and it was found that mostly coagulase negative Staphylococcus species got isolated from both the samples. Pure culture of Staphylococcus species were screened for various biochemical tests like coagulase test, catalase test etc. Gram positive, coagulase negative, catalase positive and growth in mannitol salt agar reveals the presence of characteristic Staphylococcus species and its detailed antimicrobial profile was conducted for public health point of view.

\section{Introduction}

Oral microflora of snakes mostly consists of mixed microorganisms, among which the predominant two motile isolates were gram positive coagulase negative Staphylococcus species and Pseudomonas species. During the snake bite either by venomous or non venomous snake there remains always risk of bacterial contamination in the victim at the site of bite. The microbes contaminated from the mouth of snake during bite get an optimum growing environment at the puncture site causing infection and if neglected that can cause serious necrosis at the site (Abubakar et al., 2010). In snake bite 
cases the importance is given to the envenomation but the risk of getting microbial infection is generally neglected. For snake bite envenomation the only remedy is antivenom therapy. However wound infection resulting from the bacterial contamination from snake mouth has similar significance that can be avoided by the use of definite course of antibiotic.

Several studies have reported the biofilm forming capacity of the microorganisms. But there has been less study regarding the biofilm formation of bacteria isolated from snake mouth. Such bacteria can cause chronic infection at the site of wound due to the ability of the bacteria to remain protective as an aggregative form inside an extra polymeric matrix called biofilm (Kirketerp-Moller et al., 2010). There has been report of difference in sensitivity pattern between the biofilm forming and non-biofilm forming agent of Staphylococcus species in disk diffusion tests (Mahami et al., 2010). Hence in the present study by the use of modified media the sensitivity pattern was observed of both biofilm forming as well as non-biofilm forming staphylococci isolated from mouth of Indian cobra snake to provide proper and appropriate antibiotic for healing of the wound.

\section{Materials and Methods}

In the present study two oral swabs were collected from two Indian cobra snakes of 5 year old female and 8 year old male that were presented to Teaching Veterinary Clinical Complex, OUAT, Bhubaneswar for routine therapeutic management and treatment during the period of from December 2019 to January 2020 (Fig. 1). The snakes were handled carefully throughout the exercise with the help of experts/snake handlers. The snake handler opened the mouth of snake cautiously. The collection of two oral swab samples were done by the sterile cotton tipped swab sticks (sterile, Hiculture collecting device). The swabs were gently rotated on the mucosal surface of the mouth without causing any harm or injury to the mouth of snake for the purpose of sample collection.

For bacterial isolation the swab samples were inoculated in standard broth such as Brain Heart Infusion (BHI) broth and incubated at $37^{\circ} \mathrm{C}$ for a period of $18-24$ hours. Besides BHI, the solid media used were Mannitol Salt Agar (MSA), Blood agar (5-10\% sheep blood), Mueller Hinton Agar(MHA), congo red agar, bentonite clay agar. All the media used in the study were procured from Himedia, Mumbai. The above media were prepared by rehydrating the dehydrated powdered media obtained from Himedia as per instructions labelled by the manufacturer, were autoclaved, cooled and appropriate amount of blood was added in case of blood agar preparation.

The enriched samples from BHI broth were aseptically streaked on various selective media like Mannitol Salt Agar (MSA), blood agar plate and incubated for 24 hours at $37^{\circ} \mathrm{C}$ aerobically. Single colony on the blood agar plate was examined for the pattern of haemolysis and the isolated colony was again streaked on MSA for the confirmation. The identification of the isolates were carried on by the morphological characteristics of the colony on selective media, gram's staining as well as the biochemical tests. Pure cultures of the isolates were obtained by streaking again and stored on nutrient agar slant in the temperature of $4-8^{\circ} \mathrm{C}$.

The pure cultures of staphylococci were streaked on the congo red agar plate for detection of biofilm formation (Freeman et al., 1989). The medium was prepared by mixing Brain Heart Infusion broth (37 gms/L), sucrose $(50 \mathrm{gms} / \mathrm{L})$, agar no.1 
$(10 \mathrm{gms} / \mathrm{L})$ and congo red stain $(0.8 \mathrm{gms} / \mathrm{L})$. Congo red stain was prepared separately as a concentrated aqueous solution and was autoclaved. When the agar media was cooled to $55^{\circ} \mathrm{C}$ the prepared congo red stain was added. After inoculation of the prepared plates they were incubated at $37^{\circ} \mathrm{C}$ for 24 hours. Biofilm forming organisms will produce black colour colony with crystalline consistency whereas non biofilm forming or weak slime producers will produce pink colour colony with occasional darkening at the centre. This process was repeated three times (Mathur et al., 2006).

The tissue culture plate assay is considered to be the standard test for the purpose of detection of biofilm formation (Christensen et al.1985). The pure culture of staphylococci was inoculated in BHI broth with $2 \%$ sucrose and incubated and diluted 1in 100 with sterile saline. 96 wells flat bottom tissue culture plate was inoculated with the $0.2 \mathrm{ml}$ of diluted culture individually and sterile BHI broth was used as negative standard and was incubated at $37^{\circ} \mathrm{C}$ for 24 hours. After completion of incubation period the wells were washed with $0.2 \mathrm{ml}$ of PBS to remove free floating bacteria. But the biofilm forming bacteria would remain adherent to the bottom and that were fixed with $2 \%$ sodium acetate and stained with $0.1 \%$ crystal violet. Excess stain was washed with deionized water and was kept for drying. Adherent staphylococci were uniformly stained with crystal violet.

To analyse the antibiotic sensitivity profile of biofilm forming staphylococci isolates, $0.5 \%$ bentonite clay was added as a substrate to Mueller-Hinton Agar (MHA) whereas for non biofilm forming isolates normal MHA was used without addition of bentonite clay as substrate. The pure culture was made to inoculum by diluting with normal saline to produce turbity equal to $0.5 \mathrm{McFarland}$ turbidity standard and was swabbed evenly on normal MHA for non biofilm formation and on MHA+ $0.5 \%$ bentonite for biofilm formation. The antibiotic discs used wereLinezolid (30 mcg), Levofloxacin (5mcg), Gentamicin (10mcg), Clindamycin (2mcg), Erythromycin (15mcg), Tetracycline (30 mcg), Vancomycin (30mcg), Amoxyclav (30 mcg), Ciprofloxacin $(5 \mathrm{mcg})$. All the antibiotic discs were obtained from Himedia, Mumbai. The Mueller-Hinton Agar (MHA) plates were incubated aerobically for $18-24$ hours at $37^{\circ} \mathrm{C}$ and the results were analysed as per the guidelines of Clinical and Laboratory Standards Instititute (CLSI) (Wayne 2009).

\section{Results and Discussion}

Staphylococcus species isolates were determined as per the method of Bergey's determinative bacteriology. Two yellow coloured isolates on Mannitol Salt Agar plate from two samples were identified as Staphylococcus species by morphological character and positive gram staining character (Fig. 2 and 3) and was further proved to be coagulase negative staphylococcus by performing further tests. These isolates were also subjected to biofilm production in Congo Red Agar plate and tissue culture plate as per the procedure given by Freeman et al., 1989 and Christensen et al., 1985. Two isolates showed formation of biofilm on both Congo Red Agar plate and tissue culture plate. In congo red agar the black coloured crystal like growth of staphylococci was obtained. The antibiotic sensitivity profile revealed that the zone of inhibition of biofilm producing Staphylococcus spp. (using bentonite clay on MHA) against all the 9 antibiotics was found to be less than its nonbiofilm forming agents (without use of bentonite clay on MHA) (Table 1). The average zone of inhibition (mm) for staphylococci isolate on MHA (without bentonite clay) for the following antibiotics: Linezolid (30 mcg), Levofloxacin (5mcg), Gentamicin (10mcg), Clindamycin 
(2mcg), Erythromycin (15mcg), Tetracycline (30 mcg), Vancomycin (30 mcg), Amoxyclav (30 mcg), Ciprofloxacin (5mcg) were 25, 24.3, 18.2, 23, 23.2, 24.1, 19, 16, 22.2 respectively. For the same antibiotics the average zone of inhibition shown by staphylococci isolate on
MHA (with bentonite clay) were 23, 22.8, $16.9, \quad 20.8, \quad 20, \quad 22, \quad 16.5, \quad 12, \quad 19.8 \mathrm{~mm}$ respectively. Hence it was revealed that biofilm forming staphylococci showed higher resistance towards antibiotics as compared to the non biofilm forming counterpart.

Table.1 Antibiotic sensitivity profile of Staphylococcus aureus isolate

\begin{tabular}{|r|c|c|c|c|}
\hline Sl. & Name of antibiotic & $\begin{array}{c}\text { Conc. } \\
\text { No. }\end{array}$ & & \multicolumn{2}{|c|}{ Zone of inhibition (mm) } \\
\cline { 5 - 5 } & & & $\begin{array}{c}\text { Without addition of } \\
\text { bentonite clay } \\
\text { (nonbiofilm forming) }\end{array}$ & $\begin{array}{c}\text { With addition of } \\
\text { bentonite clay } \\
\text { (biofilm forming) }\end{array}$ \\
\hline $\mathbf{1}$ & Linezolid & 30 & 25 & 23 \\
\hline $\mathbf{2}$ & Levofloxacin & 5 & 24.3 & 22.8 \\
\hline $\mathbf{3}$ & Gentamicin & 10 & 18.2 & 16.9 \\
\hline $\mathbf{4}$ & Clindamycin & 2 & 23 & 20.8 \\
\hline $\mathbf{5}$ & Erythromycin & 15 & 23.2 & 20 \\
\hline $\mathbf{6}$ & Tetracycline & 30 & 24 & 22 \\
\hline $\mathbf{7}$ & Vancomycine & 30 & 19 & 16.5 \\
\hline $\mathbf{8}$ & Amoxyclav & 30 & 16 & 12 \\
\hline $\mathbf{9}$ & Ciprofloxacin & 5 & 22 & 19.8 \\
\hline
\end{tabular}

Fig.1 Oral swabbing of Indian cobra snake

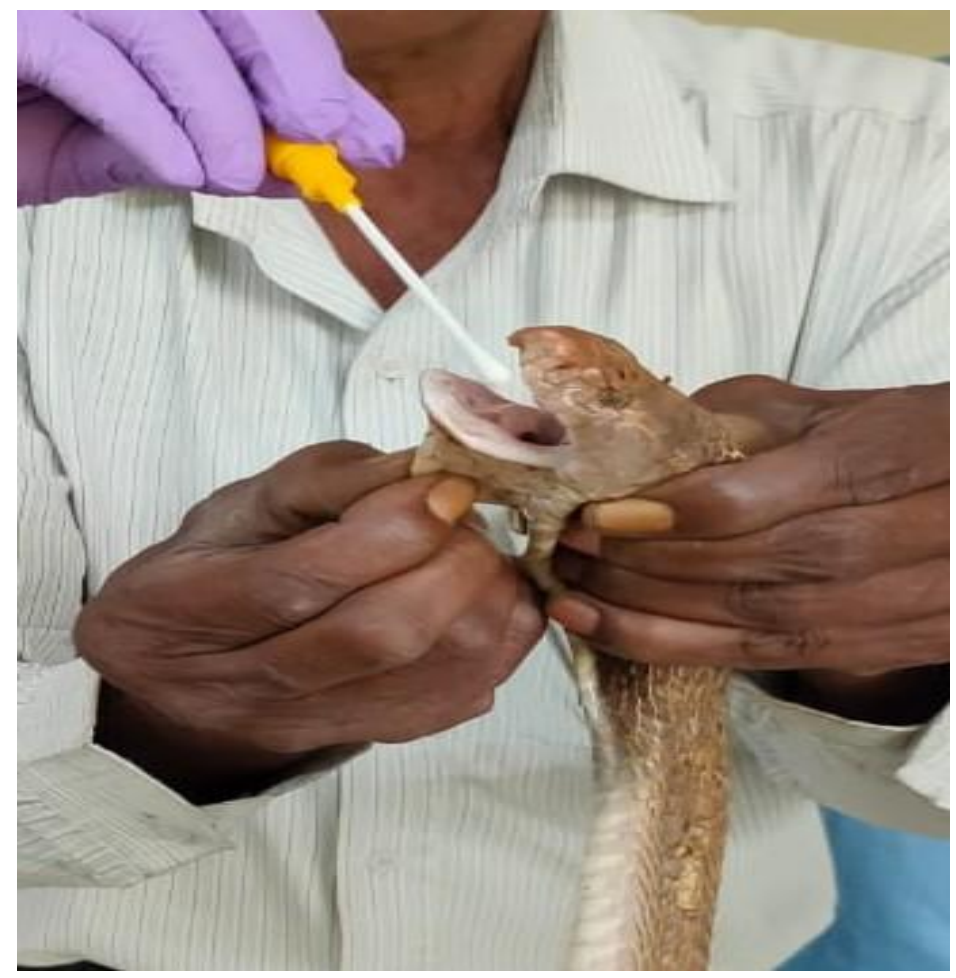


Fig.2 Golden yellow colour colony in MSA

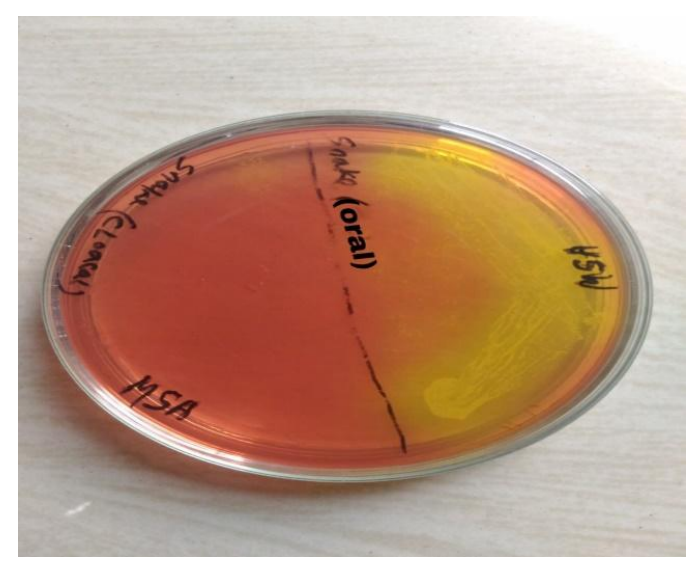

Fig.3 Gram positive staphylococci 100X

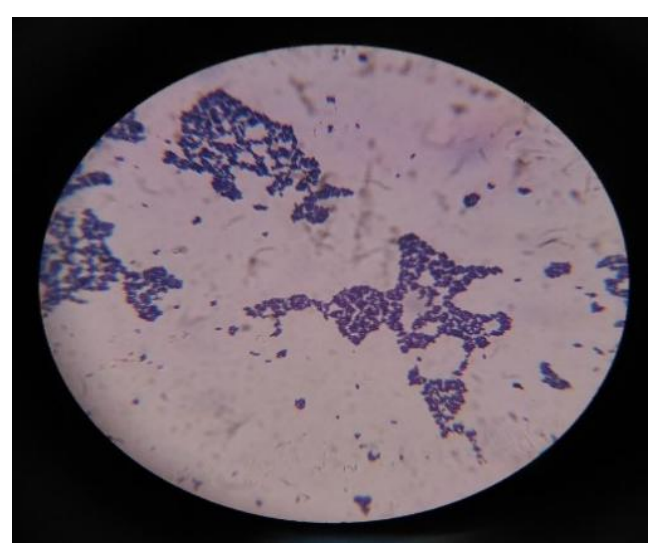

The antibiotic resistance patterns of the Staphylococcus isolates shows that both biofilm and nonbiofilm forms of Staphylococcus aureus are highly sensitive to linezolid, levofloxacin and gentamicin. Incase of clindamycin and erythromycin the non biofilm forming bacteria showed sensitivity where as the biofilm forming patterns showed little resistance. The finding of isolate from snake mouth showing sensitivity towards linezolid and gentamicin is also in agreement with results of other researchers (Shakih et al., 2017; Panda et al., 2018). However in case of all the antibiotics it was observed that the biofilm forming strains showed more resistance as compared to the non biofilm forming strains. The result is in agreement with the finding of earlier researchers
(Mahami et al., 2010; Balcázar et al., 2015; Frieri et al., 2017) who also reported the varying antibiotic sensitivity pattern against biofim forming and nonbiofilm forming isolates from different specimen. In case of snake bite wounds such biofilm forming contaminants can aggravate the condition as the antibiotic may not be effective against it as the extracellular polymeric substance (EPS) secreted by biofilm forming bacteria can act as barrier to prevent diffusion of antibiotics (Singh et al., 2017). Hence further studies are required for screening of biofilm forming microbes from snake mouth and their antibiotic sensitivity pattern so that proper and appropriate dosages of antibiotic can be chosen for proper and effective healing of snake bite wound. 


\section{References}

Abubakar SB, Habib AG, Mathew J (2010) Amputation and disability following snakebite in Nigeria. Trop Doct 40:114-

116.doi:10.1258/td.2009.090266

Balcázar JL, Subirats J and Borrego CM, 2015. Therole of biofilms as environmental reservoirs of antibiotic resistance. Front Microbiol, 6:1216,doi:

10.3389/fmicb.2015.01216.

Christensen GD, Simpson WA, Younger JJ, Baddour LM, Barrett FF, Melton DM, Beachey EH $J$ Clin Microbiol. 1985 Dec; 22(6): 996-1006

Freeman DJ, Falkiner FR, Keane CT.1989. New method for detecting slime production by coagulase negative Staphylococci, J Clin Patho., 42:872874.

Frieri M, Kumar K and Boutin A, 2017. Antibiotic resistance. J Infect Public Heal, 10: 369-378.

Kirketerp-Møller, Klaus and Zulkowski, Karen \& James, Garth. (2010). Chronic Wound colonization, Infection, and Biofilms. 10.1007/978-1-4419-60849_2.

MahamiT, Adu-Gyamfi A and Owulah C, 2010 .Comparative susceptibility of in vitrobiofilm and planktonic cells of Staphylococcus aureus to antimicrobials African J MicrobiolRes, 4(12): 1209-1214.

Mathur T, Singhal S, Khan S, Upadhyay D J, Fatma T, Rattan A.2006. Detection of biofilmformation among the clinical isolates of staphylococci: An evaluation of three different screening methods. Indian $\mathrm{J}$ Med Microbiol 2006; 24:25-9

Panda SK, Padhi L and Sahoo G. 2018. Oral bacterial flora of Indian cobra (Najanaja) and their antibiotic susceptibilities, Heliyon 4 e01008.doi: 10.1016/j.heliyon.2018.e01008

Shaikh IK, Dixit PP, Pawade BS, Lele MP and Kurhe BP. 2017. Assessment of cultivable oral bacterialflora from important venomous snakes of India and their antibiotic susceptibilities, Curr. Microbiol., 74(11): 1278-1286.

Singh S, Singh SK, Chowdhury I and Singh R, 2017.Understanding the mechanism of bacterial biofilms resistance to antimicrobial agents. The Open Microbiol J, 11: 53-62

Wayne PA, 2009. Clinical and Laboratory Standards Institute (CLSI) Performance Standards for Antimicrobial Disk Diffusion Susceptibility Tests. 19th edn., approved standard CLSI document., M100-S19.

\section{How to cite this article:}

Soumya Ranjan Khatua, Rajashree Mishra, Indramani Nath, Debiprasanna Das, Bhabani Shankar Satapathy, Kailash Singh Bisht and Iswar Anand Senapati. 2020. Study of Antimicrobial Resistance Profile of Coagulase Negative Staphylococcus Species Isolated from the Oral Cavity of Indian Cobra (Naja naja). Int.J.Curr.Microbiol.App.Sci. 9(09): 510-515. doi: https://doi.org/10.20546/ijcmas.2020.909.064 